\title{
Arbeitswelt 4.0 und Smart Machines: Augmentation als Herausforderung für die Personalentwicklung
}

\author{
Christoph Meier • Sabine Seufert $\mathbb{D} \cdot$ Josef Guggemos
}

Eingegangen: 1. April 2019 / Angenommen: 8. Juli 2019 / Online publiziert: 24. Juli 2019

(C) Springer Fachmedien Wiesbaden GmbH, ein Teil von Springer Nature 2019

Zusammenfassung Mit der zweiten Welle der Digitalisierung werden Personalentwickler neu herausgefordert. Smart Machines können bereits heute viele anspruchsvolle Verrichtungen ausführen und sie werden kontinuierlich besser. Die damit verbundenen Veränderungen werden zu oft unter dem Aspekt der Substitution von Arbeitskräften diskutiert und zu wenig unter dem Aspekt der Augmentation, d.h., im Hinblick auf das Zusammenwirken von Menschen und ,intelligenten“ Maschinen „Hand in Hand“.

Eine Diskussion zu den Folgen dieser Veränderungen findet häufig nicht statt, weil eine Verunsicherung der Belegschaft befürchtet wird. Das Konzept der Augmentationsstrategien bietet hier Orientierung und erleichtert die Diskussion - weil es aufzeigt, dass man diesen Veränderungen nicht hilflos ausgeliefert ist, sondern dass verschiedene Strategien für die Weiterentwicklung möglich sind.

Augmentation und Augmentationsstrategien sind ein geeigneter Orientierungsrahmen, um die Aufgaben für Personalentwickler zu strukturieren. Auf Augmentation ausgerichtete Personalentwicklung erfordert einen Gesamtprozess, der verschiedene Arbeitsstränge integriert: die Analyse von Veränderung bei Prozessen, bei Aufgabenzuschnitten und bei Kompetenzerfordernissen; die Gestaltung von Entwicklungsangeboten; begleitendes Veränderungsmanagement und Entwicklungsbegleitung; und schliesslich die Erfolgsbestimmung und Wirkungsüberprüfung. Bei all diesen Strängen ist eine enge Zusammenarbeit mit den jeweiligen Fachabteilungen erforderlich.

Die Umsetzung dieses Gesamtprozesses erfordert geeignete Arbeitsinstrumente. Beispielsweise zur Standortbestimmung von Beschäftigtengruppen, zur Analyse sich verändernder Aufgaben-Anforderungssysteme, oder zur Augmentations-orientierten Entwicklungsplanung. Es braucht aber auch Personalentwickler, die sich (1) mit fortgeschrittener Digitalisierung auskennen und die (2) Programme für die verschie-

C. Meier $\cdot$ S. Seufert $(\bowtie) \cdot$ J. Guggemos

Institut für Wirtschaftspädagogik, Universität St. Gallen, Guisanstrasse 1a, 9010 St. Gallen, Schweiz

E-Mail: christoph.meier@unisg.ch 
denen Augmentationsstrategien entwickeln und glaubwürdig umsetzen können nicht zuletzt auch dadurch, dass sie diese Strategien in ihrem eigenen Arbeitsfeld selbst leben.

Schlüsselwörter Künstliche Intelligenz · Smart Machines · Substitution von Arbeitskraft · komplementäre Kompetenzen · Personalentwicklung ·

Augmentation · Augmentationsstrategien · Beschäftigtengruppen ·

Prozessgestaltung

\section{Digital Transformation and Smart Machines: Augmentation as Challenge for People Development}

Abstract Digital transformation poses new challenges for people development. Today, smart machines can already perform tasks that before could be performed only by humans - and they are becoming more powerful continually. These developments are usually discussed with an eye on the substitution of (parts of) the workforce. We propose to focus on augmentation instead, i.e. on the collaboration of humans and smart machines.

Often, the changes that are related to digital transformation are not sufficiently discussed in businesses and organizations for fear of creating unease, uncertainty and frustration. The concept of augmentation strategies provides orientation and facilitates discussion. The concept helps see that humans are not subject to developments they cannot influence but rather that there are different options and strategies for development.

Augmentation and augmentation strategies are a useful framework for structuring the tasks of specialists engaged in people development. People development oriented by augmentation and augmentation strategies requires a coherent process that integrates different strands of activity: the analysis of changes in processes, in work tasks and roles as well as in competency requirements; the design of development programs; change management activities and support in development activities; and, finally, measurement of success and impact. All these strands of activity require close cooperation of people development specialists with neighboring units and the business.

The realization of this coherent process requires a specific set of tools. Tools that help determine the status quo with a particular job family, that support the analysis of changing processes and work tasks, or tools that help draw up augmentation-oriented development plans. In addition to tools, however, people development specialists are required that are knowledgeable about digital transformation and that are able to draft and implement augmentation-oriented development - not the least because they are making use of augmentation strategies in their own field of work.

Keywords artificial intelligence - smart machines - substitution of workforce · complementing competences $\cdot$ people development $\cdot$ augmentation $\cdot$ augmentation strategies $\cdot$ occupational groups $\cdot$ process design 


\section{1 ,Intelligente“ Maschinen im Arbeitsfeld}

Aktuell erleben wir eine ,zweite Welle der Digitalisierung“: neben die bereits etablierten Technologien für maschinenlesbare Daten (Technologien, mit denen Daten erfasst, gespeichert, übertragen und verarbeitet werden können) treten zunehmend Technologien, die Daten verstehen, veredeln und aktiv nutzen können (künstliche Intelligenz und maschinelles Lernen) (Wahlster 2017).

Systeme und Maschinen mit künstlicher Intelligenz (wir sprechen in der Folge von „Smart Machines“) können bereits heute viele Verrichtungen durchführen, für die in der Vergangenheit menschliche Arbeitsleistung nötig war. Dies befeuert die Debatte um die Zukunft von Arbeit und Beschäftigung in modernen Wirtschaftsgesellschaften. Im Vordergrund stehen dabei in der Regel Fragen danach, welche Beschäftigtengruppen wie betroffen sind und wann welche Beschäftigtengruppen substituiert (d.h., überflüssig) werden. Der Aspekt der Substitution von Arbeitskraft durch Smart Machines dominiert die Diskussion bisher (z. B. Frey und Osborne 2013; Nedelkoska und Quintini 2018). Wir plädieren für einen Perspektivwechsel und mehr Aufmerksamkeit für den Aspekt der Augmentation, das heisst, für das Zusammenwirken von Menschen und Smart Machines. Dabei gehen wir von zwei Annahmen aus: zum einen, dass in der Zukunft die Zusammenarbeit von Menschen und Smart Machines „Hand in Hand“ sehr viel häufiger stattfindet als heute. Zum anderen, dass insbesondere ein gut gestaltetes Zusammenwirken von Menschen und Smart Machines grosse Produktivitätsgewinne ermöglicht (Brugger und Kimmich 2017).

Die eben angerissenen Entwicklungen führen dazu, dass lebenslanges Lernen und dessen Unterstützung - eine zentrale Aufgabe der Personalentwicklung (Craig 1987) - an Bedeutung gewinnt. Dies gilt insbesondere auch für Beschäftigte, die von Automatisierungswellen in der Vergangenheit weniger betroffen waren, beispielsweise hochqualifizierte Wissensarbeiter und Manager (Davenport und Kirby 2016; Brynjolfsson und McAfee 2017).

Vor diesem Hintergrund stellen sich eine Reihe von Fragen, die für die Profession Personalentwicklung von grosser Bedeutung sind:

1. Welche Aufgaben können Smart Machines im Arbeitsfeld übernehmen?

2. Welche - komplementären - Kompetenzen sind auf Seiten der menschlichen Beschäftigten nötig, wenn diese Hand-in-Hand mit oder neben Smart Machines arbeiten?

3. Wie kann ein geeigneter Gesamtprozess gestaltet werden, über den die Personalentwicklung veränderte Kompetenzerfordernisse identifiziert und an Augmentationsstrategien ausgerichtete Entwicklungsangebote gestaltet?

4. Sind Personalentwickler für diese neue Aufgabe gerüstet und wie müssen sie sich ggf. selbst weiterentwickeln? 


\section{Was können Smart Machines?}

Künstliche Intelligenz (KI) bezeichnet die Realisierung von intelligentem Verhalten und den zugrundeliegenden Fähigkeiten auf Computern (Wahlster 2017). Auf der Grundlage von KI können Daten jetzt nicht mehr nur von Maschinen erfasst, gespeichert, verarbeitet und übertragen, sondern auch verstanden und veredelt werden. Kerngebiete der KI sind Mustererkennung, Lernen, Wissensrepräsentation, Wissensverarbeitung und Wissenspräsentation. Einsatzfelder der KI sind u. a. sprachverstehende Systeme, bildverstehende Systeme, autonome Systeme, kollaborative Roboter, intelligente Trainings- und Lernsysteme sowie auch (Chat-)Bots und virtuelle Charaktere (Wahlster 2017).

KI-basierte Maschinen und Systeme können eine Reihe verschiedener Fähigkeiten realisieren (siehe Tab. 1).

KI-basierte Systeme und Maschinen werden eingesetzt bzw. erprobt in der industriellen Fertigung (z.B. Co-Bots), in der Logistik (z. B. selbstfahrende Stadtbusse), im Bereich der Sicherheits- und Überwachungsdienstleistungen (z. B. Überwachung von Parkhäusern), bei der Analyse von Dokumenten (z. B. Rechtsberatung oder Steueramt), für personenbezogene Dienstleistungen (z. B. Koch-Roboter oder persönliche Assistenten wie Alexa/Cortana/etc.) oder als Sparrings- und Trainingspartner (z. B. KI-gesteuerte virtuelle Simulationen) (Hirsch 2017).

Tab. 1 Fähigkeiten von KI-basierten Systemen. (In Anlehnung an GRID by Deloitte/Efma 2017)

\begin{tabular}{|c|c|c|}
\hline Fähigkeiten & Anwendungsfelder & Beispiele \\
\hline $\begin{array}{l}\text { (Wieder-)Er- } \\
\text { kennen und } \\
\text { Verstehen }\end{array}$ & $\begin{array}{l}\text { Transformation von Handschrift in Maschi- } \\
\text { nenschrift } \\
\text { Transformation von gesprochener Sprache in } \\
\text { Maschinenschrift } \\
\text { Erkennen von Gegenständen auf Fotos und } \\
\text { in Videos }\end{array}$ & $\begin{array}{l}\text { Automatische Überwachung einer } \\
\text { Werkstatt im Hinblick auf Zutritte und } \\
\text { Zugriffe auf Werkzeuge }\end{array}$ \\
\hline $\begin{array}{l}\text { Repetitive } \\
\text { Verrich- } \\
\text { tungen } \\
\text { ausführen }\end{array}$ & $\begin{array}{l}\text { Landwirtschaft } \\
\text { Produktion } \\
\text { Dienstleistungen }\end{array}$ & $\begin{array}{l}\text { Unterscheidung von Nutzpflanze } \\
\text { und Unkraut auf Ebene individueller } \\
\text { Pflanzen sowie selektive Applikation } \\
\text { von Unkrautvernichtungsmittel }\end{array}$ \\
\hline $\begin{array}{l}\text { Bedeutung } \\
\text { identifizie- } \\
\text { ren }\end{array}$ & $\begin{array}{l}\text { Übersetzungen von einer Sprache in eine } \\
\text { andere Sprache }\end{array}$ & $\begin{array}{l}\text { Automatische Übersetzung von Web- } \\
\text { seiten }\end{array}$ \\
\hline $\begin{array}{l}\text { Analysieren } \\
\text { und ent- } \\
\text { scheiden }\end{array}$ & Vermögensverwaltung & $\begin{array}{l}\text { Robo-Advisory in der Vermögensver- } \\
\text { waltung }\end{array}$ \\
\hline $\begin{array}{l}\text { Artefakte } \\
\text { produzieren }\end{array}$ & Produktion mit Losgrösse 1 & $\begin{array}{l}\text { Auf Basis eines Körperscans indivi- } \\
\text { dualisierte Schuhe herstellen }\end{array}$ \\
\hline $\begin{array}{l}\text { Lernen und } \\
\text { verbessern }\end{array}$ & Weiterentwicklung von Algorithmen & $\begin{array}{l}\text { Nicht-überwachtes, Feedback-basier- } \\
\text { tes Lernen beim Schachprogramm } \\
\text { AlphaZero (Klein 2017) }\end{array}$ \\
\hline
\end{tabular}




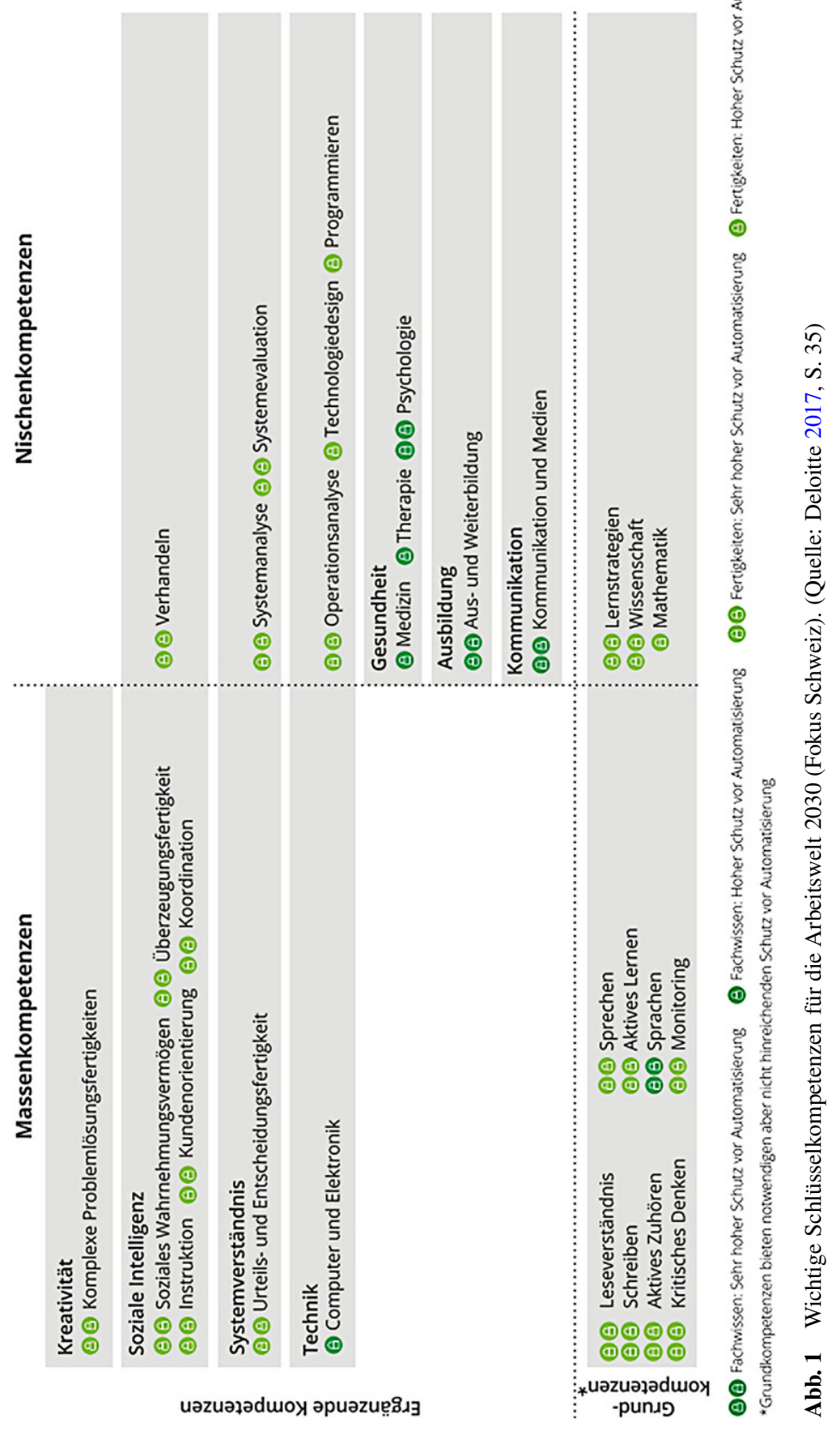




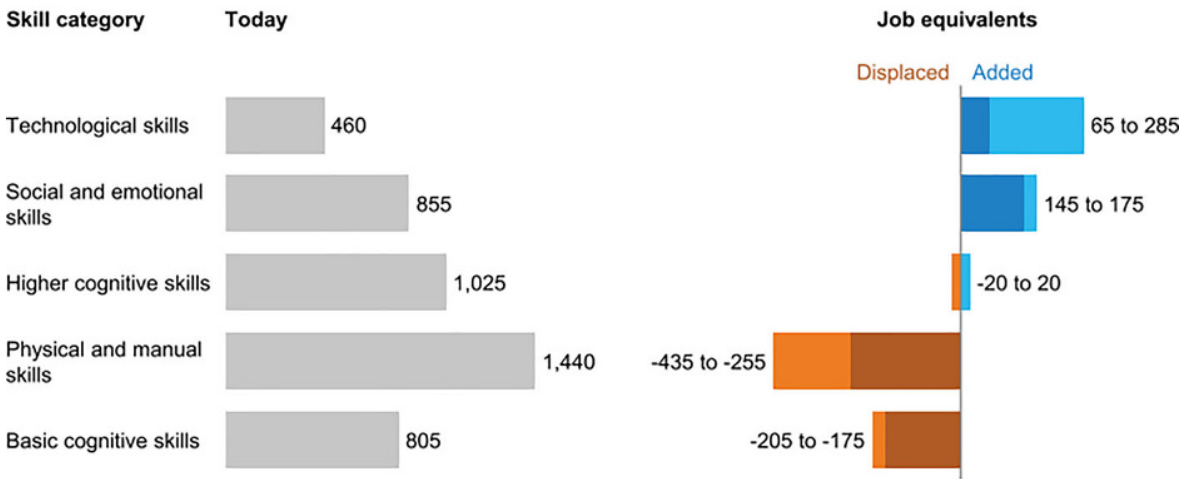

NOTE: Calculation based on evaluation of change in people employed in an occupation from A future that works: Automation, employment, and productivity. McKinsey Global Institute, January 2017.

Abb. 2 Veränderungen bei der Nachfrage nach Kompetenzen für den Wirtschaftsraum Schweiz bis 2030. (Quelle: Bughin et al. 2018, S. 15)

\section{Welche Kompetenzen benötigen Mitarbeitende künftig?}

In den letzten Jahren sind verschiedene Studien publiziert worden, die vor dem Hintergrund dieser zweiten Welle der Digitalisierung einen Blick auf künftige Kompetenzerfordernisse werfen (Deloitte 2017; McKinsey Global Institute 2018; Bughin et al. 2018). Diese Studien entwickeln Zukunftsbilder dazu, welche Kompetenzen für die Beschäftigungsfähigkeit in 2030 wichtig sind und für welche Kompetenzen sich die Nachfrage künftig eher verringern oder eher erhöhen wird.

Die Autoren der Studie von Deloitte betonen, dass künftige Beschäftigungsfähigkeit nicht so sehr durch einzelne Kompetenzen erreicht wird als vielmehr durch das Zusammenspiel verschiedener Fertigkeiten und die dadurch mögliche Aneignung weiterer Kompetenzen. Sie unterscheiden daher Grundkompetenzen, die die Voraussetzung für die Aneignung weiterer Kompetenzen sind. Darüber hinaus unterscheiden sie Massenkompetenzen, die für die Mehrheit der neu entstehenden Stellen (erwartet werden für die Schweiz bis zu 700.000 neue Stellen bis 2030) wichtig sind, und Nischenkompetenzen, die für weniger als die Hälfte der neu entstehenden Stellen wichtig sind (vgl. Abb. 1).

Eine etwas andere Gliederung verschiedener, zukunftsrelevanter Kompetenzbereiche nimmt eine Studie zur Zukunft der Arbeit mit Fokus auf die Schweiz vor, die von McKinsey Global Institute und McKinsey\&Company Schweiz erstellt wurde. Hier werden fünf übergreifende Kompetenzbereiche (Technologie-Kompetenzen, Soziale und emotionale Kompetenzen, Höhere kognitive Fertigkeiten, Körperliche und manuelle Fertigkeiten sowie Kognitive Grundkompetenzen, vgl. Abb. 2) und insgesamt 25 untergeordnete Kompetenzen unterschieden. Für diese Kompetenzbereiche wird zum einen aufgezeigt, wie gross die Nachfrage aktuell ist (vgl. Abb. 2, unten, lin- 


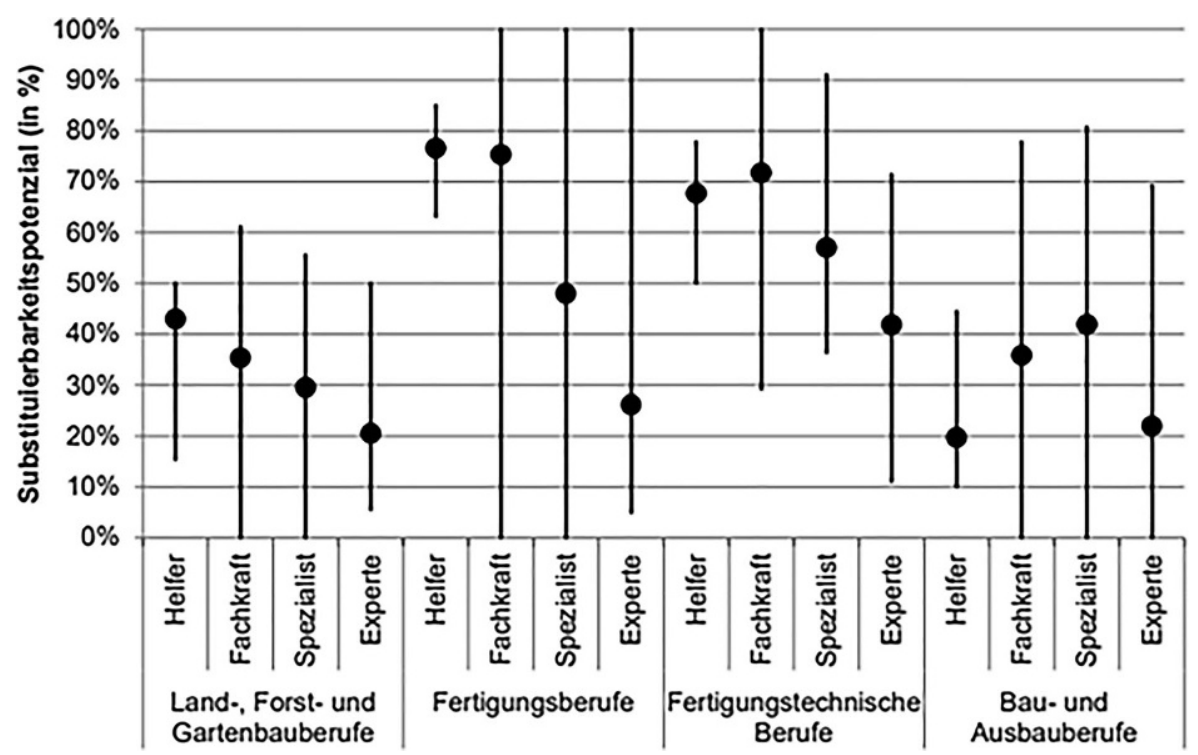

Abb. 3 Substituierbarkeit in Produktionsberufen. (Quelle: Dengler und Matthes 2015, S. 16)

ke Seite) und es werden Veränderungen in der Nachfrage bis 2030 geschätzt (vgl. Abb. 2, unten, rechte Seite).

Auch wenn solche Prognosen unsicher sind, insbesondere wenn sie sich auf die Fortschreibung von Trends der Vergangenheit stützen, so konkretisieren sie doch eine aktuell weit verbreitete Erwartung: Arbeitsaufkommen und nachgefragte Kompetenzprofile werden sich in absehbaren Zukunft deutlich verändern. Die daran anschliessenden Fragen lauten, welche Beschäftigten- bzw. Berufsgruppen davon in welcher Weise betroffen sein werden und welche Aufgaben sich daraus für die betriebliche Personalentwicklung ergeben.

Relevant ist hierzu die Studie von Dengler und Matthes (2015). Zum einen zeigt sie Unterschiede zwischen Branchen und Berufssegmenten auf, zum anderen Unterschiede zwischen Kombinationen von Berufssegmenten und Anforderungsniveaus (Helfer - Fachkraft - Spezialist - Experte), vgl. Abb. 3. Dabei wird deutlich, dass es nicht unbedingt weniger qualifizierte Helfer-Profile sind, bei denen das Risiko, durch Smart Machines ersetzt zu werden, besonders hoch ist, sondern dass dies auch Fachkräfte und Fachspezialisten betrifft.

Alle hier angeführten Studien betonen im Hinblick auf die prognostizierten Veränderungen bei den Kompetenzerfordernissen die Bedeutung von weiteren Anstrengungen im Bereich der Aus- und Weiterbildung, führen dies aber nicht konkret aus. Hier kommt aus unserer Sicht das Konzept der Augmentation und der Augmentationsstrategien zum Tragen. Wir erläutern zunächst diese beiden Konzepte und skizzieren dann einen daran orientierten Gesamtprozess für Personalentwicklung in Unternehmen und Organisationen. 


\section{Augmentation und Augmentationsstrategien als Orientierungsrahmen für die Personalentwicklung}

\subsection{Augmentation und Augmentationsstrategien}

Augmentation bedeutet Verbesserung und bezeichnet, in Abgrenzung zu Substitution bzw. Verdrängung, das produktive Zusammenwirken von Menschen und ,intelligenten“ Maschinen. Ein hilfreiches Konzept für die Formulierung und Ausarbeitung von Personalentwicklungsstrategien im Zeitalter von Smart Machines ist aus unserer Sicht das Konzept der Augmentationsstrategien (Davenport und Kirby 2016). Davenport und Kirby fokussieren die Folgen des Eindringens von Smart Machines in die Arbeitsfelder von hoch qualifizierten Wissensarbeitern und unterscheiden dabei fünf Strategien für die Weiterentwicklung (siehe Tab. 2).

Davenport und Kirby zufolge sind diese Strategien nicht an spezifische Berufsgruppen oder Professionen gebunden sind. Die Beispiele in Tab. 3 verdeutlichen dies.

\subsection{Unterschiedliche Beschäftigtengruppen}

Davenport und Kirby haben in ihrem Buch „Only humans need apply“ den Blick auf hochqualifizierte Wissensarbeiter gerichtet. Das von ihnen entwickelte Konzept der fünf Augmentationsstrategien kann aber auch auf andere Beschäftigtengruppen angewendet werden. Das scheint aus unserer Sicht auch geboten, um der Personalentwicklung ein wirksames Arbeitsinstrumentarium zur Bewältigung der Folgen von Digitalisierung an die Hand zu geben.

Im Rahmen eines laufenden Entwicklungsprojekts (,scil Innovationskreis Augmentation“, Meier 2018b) entwickeln wir gemeinsam mit Praxispartnern auf Augmentation bezogene Werkzeuge für die Personalentwicklung. Im Rahmen der gemeinsamen Arbeit hat sich u.a. gezeigt, dass das von Davenport und Kirby vorgeschlagene Rahmenmodell nicht nur für hoch qualifizierte Beschäftigtengruppen sinnvoll genutzt werden kann.

Davenport und Kirby gehen allerdings - vielleicht etwas idealistisch - davon aus, dass die Beschäftigten selbst entscheiden (müssen), welche der möglichen Augmentationsstrategien sie verfolgen. Und sie sehen die verschiedenen Entwicklungsstrategien als mehr oder weniger gleich wichtige Optionen nebeneinander. In der Unternehmenspraxis stellt sich dies häufig anders dar. So kann beispielsweise die Leitung des Vertriebs einer Bank vorgeben, welche digitalen, KI-basierten Assistenten von den Anlageberatern eingesetzt werden müssen. Für die Anlageberater bleibt in einer so geführten Organisation nur die Option „Step in“ oder aber der Wechsel auf eine andere Stelle - innerhalb oder ausserhalb der Bank. Das gleiche kann beispielsweise für Mitarbeitende im Call-Center gelten. Hier steht also „Step in“ als Entwicklungsoption für die Beschäftigten ganz klar im Vordergrund - wenn es nicht gar die einzige angebotene Option ist. Andere Unternehmen verfolgen diesbezüglich möglicherweise eine andere Personalpolitik und gewähren ihren Mitarbeitenden mehr Entscheidungsspielraum, um auch andere Entwicklungsstrategien in Betracht zu ziehen bzw. eine für sie selbst passende Augmentationsstrategie zu verfolgen. 


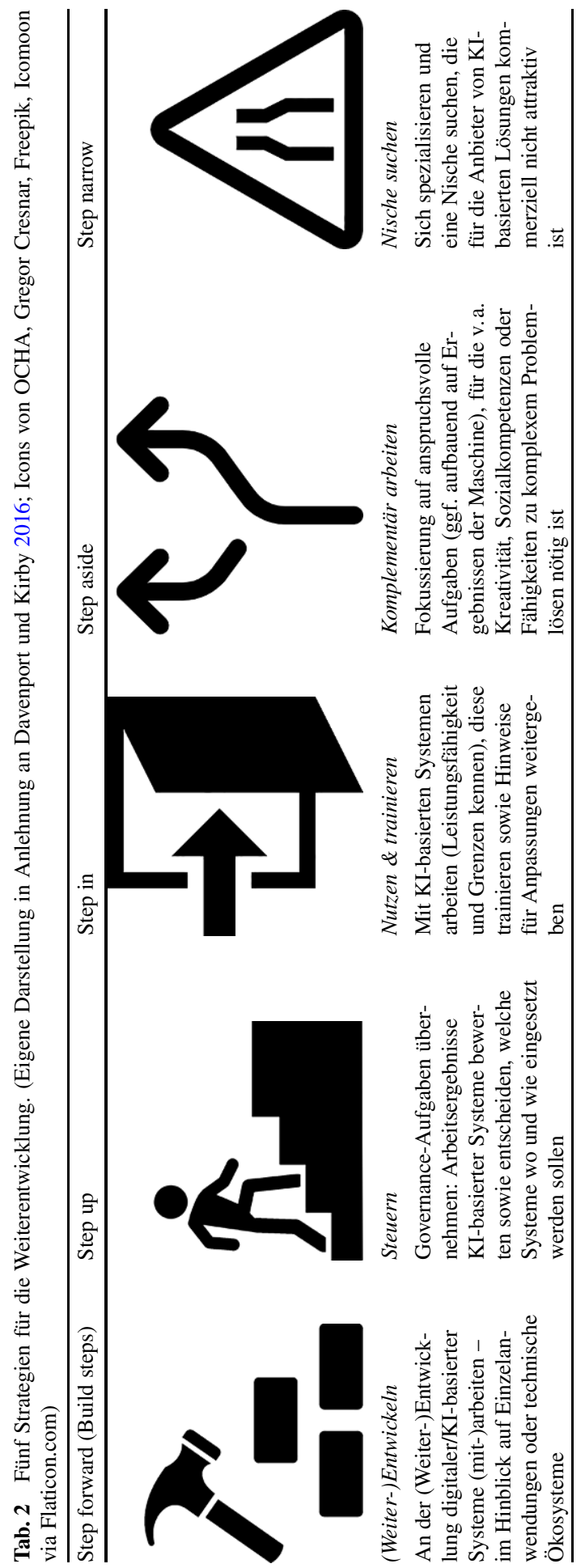


Tab. 3 Entwicklungsoptionen und Beispiele für Berufsgruppen. (Nach Davenport und Kirby 2016)

\begin{tabular}{|c|c|c|c|}
\hline & Rechtsanwalt & Finanzdienstleister & Marketing-Experte \\
\hline $\begin{array}{l}\text { Step } \\
\text { forward } \\
\text { (build) }\end{array}$ & $\begin{array}{l}\text { Mitarbeit an Entwicklung von } \\
\text { Systemen für E-Discovery, } \\
\text { Patentprüfung etc }\end{array}$ & $\begin{array}{l}\text { Mitarbeit an Entwicklung } \\
\text { von digitalen Kundenbe- } \\
\text { ratungs-Systemen }\end{array}$ & $\begin{array}{l}\text { Mitarbeit an Entwicklung } \\
\text { von Algorithmen zur Opti- } \\
\text { mierung von Werbeplätzen }\end{array}$ \\
\hline Step up & $\begin{array}{l}\text { Entscheiden, dass E-Disco- } \\
\text { very Werkzeuge eingesetzt } \\
\text { werden, um Gerichtsverfahren } \\
\text { vorzubereiten }\end{array}$ & $\begin{array}{l}\text { Bereiche identifizieren, } \\
\text { die mehr/bessere Auto- } \\
\text { matisierung benötigen }\end{array}$ & $\begin{array}{l}\text { Einsatz digitaler Systeme } \\
\text { für Brand-Management } \\
\text { orchestrieren }\end{array}$ \\
\hline Step in & $\begin{array}{l}\text { Expertise in automati- } \\
\text { sierter Auswertung von } \\
\text { (Vertrags-)Dokumenten auf- } \\
\text { bauen (E-Discovery) }\end{array}$ & $\begin{array}{l}\text { Expertise im Bereich } \\
\text { Online-Beratung und } \\
\text { Online-Unterstützung von } \\
\text { Kunden aufbauen }\end{array}$ & $\begin{array}{l}\text { Expertise zu automatisier- } \\
\text { ter Preisbildung aufbauen; } \\
\text { beobachten und ggf. inter- } \\
\text { venieren }\end{array}$ \\
\hline $\begin{array}{l}\text { Step } \\
\text { aside }\end{array}$ & $\begin{array}{l}\text { Fokussierung auf Vertrieb, } \\
\text { Kunden-Mgmt, Kundenbera- } \\
\text { tung in einer Kanzlei }\end{array}$ & $\begin{array}{l}\text { Fokussierung auf Kom- } \\
\text { munikation mit Kunden }\end{array}$ & $\begin{array}{l}\text { Fokussierung auf Kreativ- } \\
\text { arbeit und Kundenberatung }\end{array}$ \\
\hline $\begin{array}{l}\text { Step } \\
\text { narrow }\end{array}$ & $\begin{array}{l}\text { Fokussierung auf Regulie- } \\
\text { rung in einem Sektor/einer } \\
\text { Branche, die vorerst nicht } \\
\text { automatisiert wird }\end{array}$ & $\begin{array}{l}\text { Fokussierung auf Kun- } \\
\text { densegmente, wo wenig/ } \\
\text { nicht automatisiert wird }\end{array}$ & $\begin{array}{l}\text { Fokussierung auf Nutzung } \\
\text { von Werbeflächen im } \\
\text { öffentlichen Raum }\end{array}$ \\
\hline
\end{tabular}

\subsection{Prozessgestaltung}

Eine am Konzept der Augmentation und der Augmentationsstrategien orientierte Personalentwicklung erfordert einen entsprechend gestalteten Gesamtprozess. Das Konzept und einzelne Bausteine dieses Gesamtprozesses wurden im Rahmen des bereits erwähnten Innovationskreises erarbeitet. Dabei erfolgte eine zirkuläre Bewegung über verschiedene Entwicklungsstufen und Reviews in der Arbeitsgruppe hinweg (vgl. Abb. 4). Dieses Vorgehen wurde sowohl bei der Entwicklung einzelner Instrumente (z. B. Interviewleitfaden zur Standortbestimmung) als auch bei der Entwicklung des Gesamtmodells umgesetzt.

Dieser Gesamtprozess umfasst fünf Bereiche:

1. Beteiligte Anspruchsgruppen

2. Analyse und Kompetenzentwicklung

3. Veränderungsmanagement

4. Erfolgsbestimmung/Wirkungsprüfung

5. Werkzeuge und Arbeitshilfen

Wer in diesen Gesamtprozess eingebunden werden sollte, kann sich je nach Unternehmenskontext unterscheiden. Relevante Anspruchsgruppen sind unter anderen das (Top-)Management, verschiedene HR-Bereiche (inkl. Learning \& Development/ Personalentwicklung), die Mitarbeitenden und deren Vorgesetzte, der Betriebsrat bzw. die Personalkommission.

Im Hinblick auf den Arbeitsstrang „Analyse und Kompetenzentwicklung“ stehen die folgenden Aufgaben im Vordergrund (vgl. Abb. 5): 
Abb. 4 Iteratives Vorgehen bei der Entwicklung von Gesamtkonzept und einzelnen Bausteinen. (Quelle: eigene Darstellung)

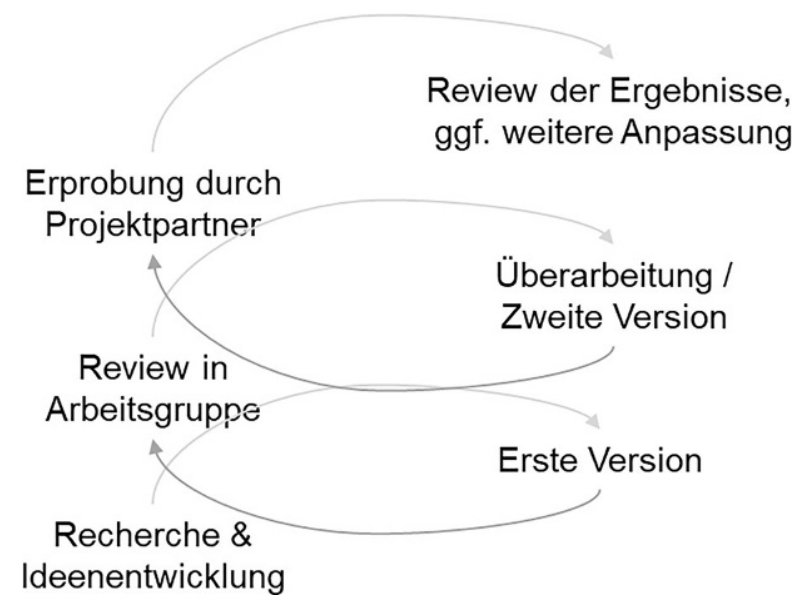

- Klären der mit dem Einsatz einer neuen Smart Machine (bzw. allgemeiner, einer neuen technischen Lösung) verbundenen Veränderungen bei Aufgaben einerseits und Kompetenzerfordernissen andererseits. Welche Aufgaben kommen neu hinzu, fallen weg oder verändern sich im Umfang?

- Welche Anforderungen ergeben sich aus diesen Veränderungen im Hinblick auf Kompetenzbereiche und Kompetenzausprägungen?

- Was sind relevante bzw. realistische Augmentationsstrategien? Wird eine Augmentationsstrategie priorisiert oder vorgegeben? Gibt es ergänzende Augmentationsstrategien oder Wahlmöglichkeiten?

- Welche konkreten Kompetenzerfordernisse ergeben sich aus der priorisierten Augmentationsstrategie? Wie müssen sich die betroffenen Beschäftigten weiterentwickeln?

- Wie können die für die Umsetzung der priorisierten Augmentationsstrategie erforderlichen Kompetenzen effektiv und effizient entwickelt werden?

Im Zuge der Einführung ,,intelligenter“ Maschinen kann sich das Aufgabenprofil von Mitarbeitenden deutlich und gegebenenfalls sogar zunehmend verändern - insbesondere dann, wenn diese Maschinen kontinuierlich besser und leistungsfähiger werden. Vor diesem Hintergrund erlangt ein zweiter, parallellaufender Arbeitsstrang „Veränderungsmanagement“" besondere Bedeutung. Die Aufgaben in diesem Strang sind u. a. die folgenden:

- Information, Orientierung, Sensibilisierung und Onboarding der Betroffenen

- Herstellen klarer Rahmenbedingungen für den Gesamtprozess

- Hilfreich ist beispielsweise eine Leitlinie wie „gemeinsame Verantwortung“, über die sich die Gesamtorganisation zur Verantwortung bekennt, aber auch ein Entwicklungsbeitrag von den Mitarbeitenden eingefordert wird (vgl. z. B. Donovan und Benko 2016).

- Standortbestimmungen 
- Wie steht es um die Veränderungsbereitschaft der Beschäftigten im Hinblick auf die durch Smart Machines zu erwartenden Veränderungen?

- Veränderungsbilanz und Anpassungen

- Wie gestaltet sich die Kosten-/Nutzen-Bilanz der Beschäftigten durch die veränderten Aufgaben und Kompetenzerfordernisse? Welche unangenehmen Aufgaben können sie an eine Maschine abgeben? Welche wertgeschätzten Aufgaben müssen sie abgeben?

- Veränderungsbereitschaft

- Sind die betroffenen Beschäftigten bereit, für sich selbst tragfähige Augmentationsstrategien zu formulieren oder entsprechende Vorgaben anzunehmen?

- Veränderungs- und Entwicklungsbegleitung

- Sind die betroffenen Beschäftigten in der Lage, eine Augmentationsstrategie nachhaltig zu verfolgen - auch in schwierigen Phasen - und welche Unterstützung benötigen sie dabei?

Parallel zu den beiden schon genannten Strängen sind auch Aspekte der Erfolgsbestimmung und Wirkungsüberprüfung zu bearbeiten. Dazu gehören das Beachten von Quality Gates (,Ist die fokussierte Beschäftigtengruppe bereit, sich auf einen Entwicklungsprozess einzulassen?") ebenso wie das Definieren von Erfolgskriterien und das Durchführen von Wirkungsmessungen.

Ausgehend von etablierten Rahmenmodellen für die Evaluation von (Personal-) Entwicklungsprojekten (Kirkpatrick 1996; Pulliam Phillips und Phillips 2007; Kurz und Kubek 2013; Thalheimer 2018) wurde hierzu ein erweitertes Stufenmodell entwickelt. Dieses Modell unterscheidet drei übergeordnete Bereiche und bis zu zehn Evaluationsebenen. Nachfolgend Beispiele für überprüfbare Formulierungen von Erfolg auf diesen verschiedenen Ebenen:

- Output: Ergebnisse im Hinblick auf die bereitgestellten Entwicklungsangebote

- Ein Gesamtprozess für Augmentations-orientierte PE ist definiert/verfügbar.

- Die adressierte Zielgruppe ist zu den anstehenden Veränderungen orientiert.

- Die adressierte Zielgruppe lässt sich auf den Entwicklungsprozess ein.

- Outcomes: Ergebnisse im Hinblick auf die Zielgruppe

- Die adressierte Zielgruppe verfügt über Wissen, Fertigkeiten und Einstellungen, die gemäss der gewählten Augmentationsstrategie erforderlich sind.

- Die adressierte Zielgruppe erfährt ausreichend Begleitung bzw. Unterstützung im Entwicklungsprozess.

- Mitglieder der Zielgruppe bearbeiten neue Aufgaben gemäss der gewählten Augmentationsstrategie.

- Impact: Ergebnisse im Hinblick auf die Gesamtorganisation

- Werte für relevante Indikatoren (Personalplanung, betriebliche Leistung, Fluktuation, Employer Branding) ändern sich in die gewünschte Richtung.

- Die gewählte Augmentationsstrategie erweist sich auf mittlere Sicht als passend und zielführend für die Zielgruppe. 


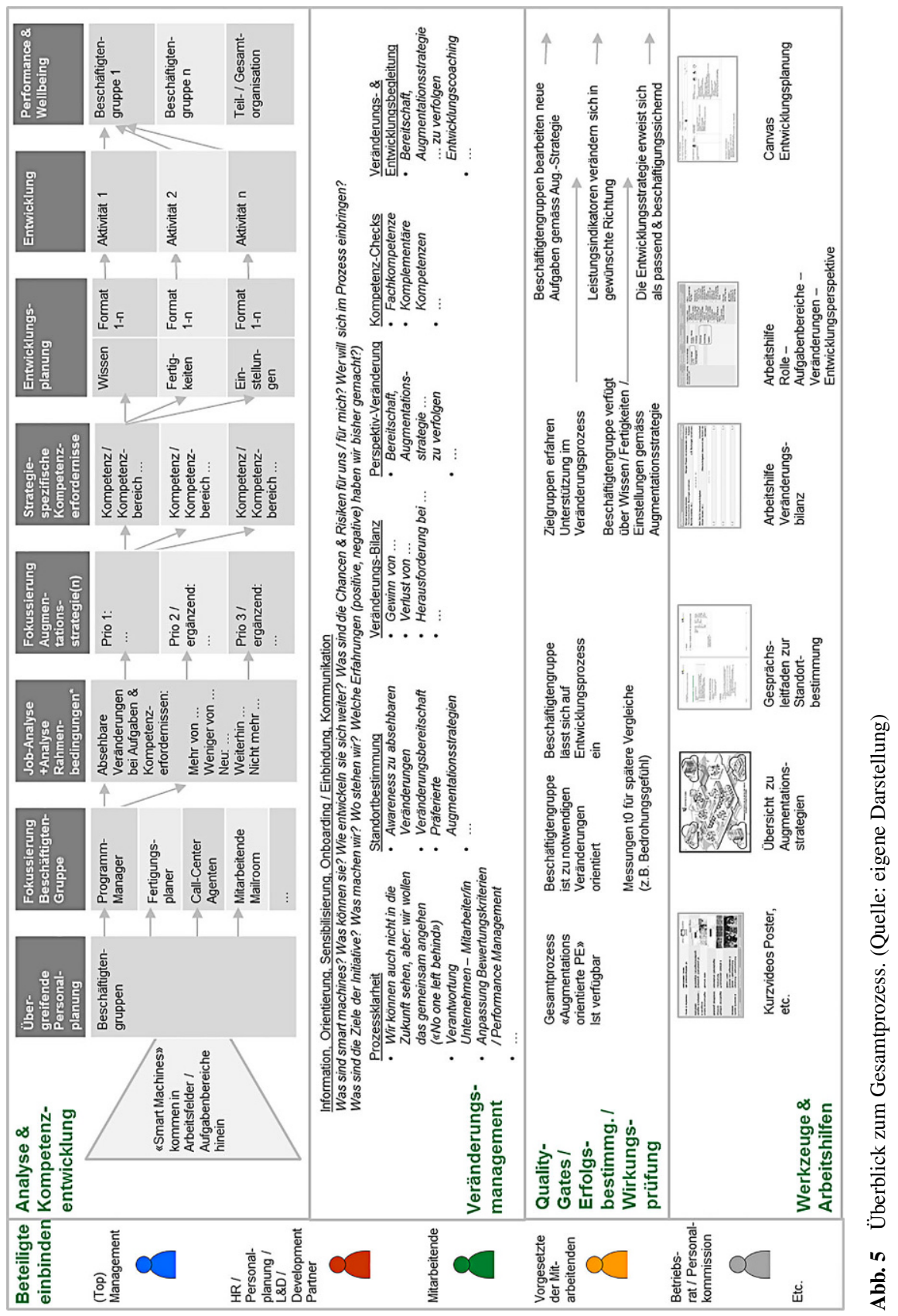




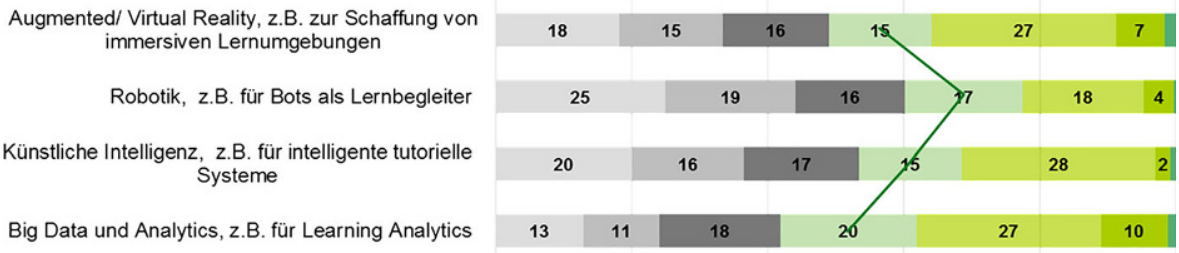

$=1$ = nicht vorhanden $=2 \| 3=4=$ neutral $\| 5=6 \approx 7=$ sehr hoch

Abb. 6 Ausprägung des Wissens von Personalentwicklern zu Aspekten fortgeschrittener Digitalisierung. (Quelle: Seufert et al. 2018, S. 11)

- Der ROI für das auf Augmentation ausgerichtete Entwicklungsprogramm ist positiv.

- Die Wahrnehmung von KI und Smart Machines durch in der Gesamtorganisation verschiebt sich von „Bedrohung“ in Richtung „Chance“.

Zur Unterstützung der Umsetzung der Arbeiten in den oben angesprochenen Strängen wurden eine Reihe von verschiedenen Arbeitshilfen entwickelt. Diese reichen von Visualisierungen zu den Augmentationsstrategien über diagnostische Instrumente bis hin zu Hilfen für die Entwicklungsplanung. Abb. 5 zeigt den in den vorangegangenen Abschnitten skizzierten Gesamtprozess in einer Übersicht. Zu sehen sind relevante Anspruchsgruppen (Beispiele, ganz links) und die parallel laufenden Arbeitsstränge. Einzelelemente sind näherungsweise dort platziert, wo sie im Umsetzungsprozess zum Einsatz kommen würden.

\section{Sind Personalentwickler bereit für die Umsetzung?}

Die Umsetzung des in diesem Beitrag skizzierten Gesamtprozesses einer an Augmentationsstrategien orientierten Personalentwicklung stellt neue Anforderungen an die Personalentwickler. Diese neuen Anforderungen betreffen beispielsweise ein Verständnis der zweiten Welle der Digitalisierung, ein Verständnis von Smart Machines und der Mechanismen, über die diese weiterentwickelt werden bzw. sich selbst weiterentwickeln, ein Verständnis für veränderte Kompetenzerfordernisse und komplementäre Kompetenzen in einer mit Smart Machines durchsetzten Arbeitswelt sowie nicht zuletzt auch ein Verständnis für die oben skizzierten Erfordernisse eines begleitenden Veränderungsmanagements.

Eine in 2018 im deutschsprachigen Raum durchgeführte Studie (Seufert et al. 2018) hat die digitalen Kompetenzen von Personalentwicklern und deren präferierte Augmentationsstrategien beleuchtet. Dabei hat sich folgendes gezeigt: Zum einen stehen Personalentwickler mehrheitlich den mit der Digitalisierung verbundenen Veränderungen positiv gegenüber. Zum anderen verfügen sie nach eigener Einschätzung über hohe Kompetenzen im Bereich Veränderungsmanagement. Dies ist ermutigend.

Allerdings ist das Wissen unter Personalentwicklern zu Aspekten fortgeschrittener Digitalisierung bislang noch eher gering ausgeprägt. Abb. 6 zeigt das Ergebnis dieser 


\begin{tabular}{|c|c|c|c|c|}
\hline $\begin{array}{l}\text { STEP-FORWARD } \\
\text { (Build) }\end{array}$ & STEP-UP & STEP-IN & STEP-ASIDE & STEP-NARROW \\
\hline $\begin{array}{c}\text { Mittelwert } \\
3.96\end{array}$ & $\begin{array}{c}\text { Mittelwert } \\
4.61\end{array}$ & $\begin{array}{c}\text { Mittelwert } \\
4.15\end{array}$ & $\begin{array}{c}\text { Mittelwert } \\
5.88\end{array}$ & $\begin{array}{c}\text { Mittelwert } \\
4.13\end{array}$ \\
\hline Rang 5 & Rang 2 & Rang 3 & Rang 1 & Rang 4 \\
\hline
\end{tabular}

Abb. 7 Rangfolge der Präferenz von Augmentationsstrategien bei Personalentwicklern. (Quelle: Seufert et al. 2018, S. 21)

Studie zum verfügbaren Wissen über Themen wie Virtual Reality, KI, Robotik und Big Data/Analytics. Die grüne Hilfslinie verweist auf die Mitte der Antwortskala. Links davon sind die Antworten aufgetragen, die für eher wenig vorhandenes Wissen stehen. Nicht nur bei dieser Frage zeigt sich, dass die Personalentwickler ihr eigenes Wissen als eher wenig stark ausgeprägt einschätzen.

Im Hinblick auf Augmentationsstrategien wird deutlich, dass die bei dieser Studie befragten Personalentwickler für sich selbst die Strategie „Step aside“ präferieren (vgl. Rang 1 in Abb. 7). Das heisst, sie sehen sich selbst primär nicht mit Smart Machines arbeiten (die auch für den Bereich Human Resources und Learning \& Development zunehmend verfügbar werden - vgl. Meier 2018a), sondern vor allem komplementär daneben (vgl. die Definition der Augmentationsstrategien in Tab. 2).

Damit sie den oben skizzierten Gesamtprozess einer an Augmentation und Augmentationsstrategien orientierten Personalentwicklung umsetzen können, sollten sich auch die Personalentwickler selbst weiterentwickeln. Sie sollten ein tieferes Verständnis der digitalen Transformation entwickeln und mehr Wissen im Bereich der fortgeschrittenen Digitalisierung aufbauen (insbesondere zu künstlicher Intelligenz und zu Smart Machines - auch im eigenen Arbeitsfeld). Sie sollten sich mit Smart Machines und KI-basierten Systemen für die Personalentwicklung vertraut machen und diese selbst einsetzen können - wo sinnvoll und zielführend. Und sie sollten das Konzept der Augmentation und der Augmentationsstrategien für sich selbst und ihren eigenen Kontext anwenden können, um davon ausgehend Programme für andere Beschäftigtengruppen gestalten und umsetzen zu können.

\section{Diskussion ermöglichen}

In vielen Unternehmen und Organisationen werden die Entwicklungen im Bereich Smart Machines und die Folgen für Beschäftigung und Kompetenzerfordernisse gegenüber den Mitarbeitenden nicht thematisiert. Dahinter steht häufig die Befürchtung, dass eine Diskussion nur zu Verunsicherung führt - insbesondere dann, wenn noch gar nicht genau Auskunft darüber gegeben werden kann, wer wann in welcher 
Form betroffen sein wird. Bei dem Versuch, das Thema zunächst einmal gedeckelt zu halten, wird aber ausgeblendet, dass die Beschäftigten sehr wohl - auch in ihrem privaten Alltag - mit diesen Themen in Berührung kommen (etwa, wenn sie zuhause digitale Assistenten wie Alexa, Google oder Siri nutzen). Ein Nicht-Thematisieren kann daher die Verunsicherung möglicherweise sogar noch erhöhen.

Das Konzept der Augmentationsstrategien bietet aus unserer Sicht nicht nur einen Orientierungsrahmen für die Personalentwicklung. Es bietet allgemein Orientierung für Führungskräfte und Beschäftigte. Es ermöglicht, die Entwicklungen im Bereich Smart Machines und die Folgen für Beschäftigung und für Kompetenzerfordernisse zum Thema zu machen. Und zwar, weil es aufzeigt, dass man diesen Veränderungen nicht macht- und hilflos ausgeliefert ist. Vielmehr gibt es verschiedene, sich gegebenenfalls ergänzende Strategien für die Weiterentwicklung - auf der Ebene der Einzelperson ebenso wie auf der Ebene von Beschäftigtengruppen. Es ist unsere Überzeugung, dass alle die in Abb. 5 gezeigten Anspruchsgruppen das Konzept der Augmentationsstrategien verstehen sollten und dass sie dieses bei ihrem Handeln permanent als Hintergrundfolie berücksichtigen sollten.

\section{Literatur}

Brugger S, Kimmich M (2017) Onboarding des Kollegen Roboter. changement 2017(3):31-34

Brynjolfsson E, McAfee A (2017) Von Managern und Maschinen. Harv Bus Manag 2017:22-34

Bughin J, Ziegler M, Mischke J, Wenger F, Reich A, Läubli D, Sen M, Schmidt M (2018) The future of work: Switzerland's digital opportunity. McKinsey\&Company. https://www.mckinsey.com/featuredinsights/europe/the-future-of-work-switzerlands-digital-opportunity

Craig RL (Hrsg) (1987) Training and development handbook; A guide to human resource development. McGraw-Hill, Berkeley

Davenport TH, Kirby J (2016) Only humans need apply; Winners and losers in the age of smart machines. Harper Business, New York

Deloitte (2017) Welche Schlüsselkompetenzen braucht es im digitalen Zeitalter?; Auswirkungen der Automatisierung auf die Mitarbeiter, die Unternehmen und das Bildungssystem. Deloitte AG, Deloitte

Dengler K, Matthes B (2015) Folgen der Digitalisierung für die Arbeitswelt; Substituierbarkeitspotenziale von Berufen in Deutschland. Institut für Arbeitsmarkt- und Berufsforschung der Bundesagentur für Arbeit. http://doku.iab.de/forschungsbericht/2015/fb1115.pdf

Donovan J, Benko C (2016) AT\&T's Talent Overhaul. Harv Bus Rev 2016:68-73

Frey CB, Osborne M (2013) The future of employment. University of Oxford. https://www.oxfordmartin. ox.ac.uk/downloads/academic/The_Future_of_Employment.pdf

GRID by Deloitte, Efma (2017) AI and you; Perceptions of Artificial Intelligence from the EMEA financial services industry. Deloitte Consulting S.r.l. / Efma. https://www2.deloitte.com/content/dam/Deloitte/ cn/Documents/technology/deloitte-cn-tech-ai-and-you-en-170801.pdf

Hirsch V (2017) TEDx Manchester: AI \& the future of work (Video). Blogpost. http://vhirsch.com/blog/ 2017/03/22/tedx-manchester-ai-the-future-of-work-video/.

Kirkpatrick DL (1996) Evaluating training programs. The four levels. Berrett-Koehler, San Francisco

Klein M (2017) Google's AlphaZero besiegt Stockfish in einem 100 Partien Vergleich. https://www.chess. com/de/news/view/google-s-alphazero-besiegt-stockfish-in-einem-100-partien-vergleich-3971

Kurz B, Kubek D (2013) Kursbuch Wirkung; Das Praxishandbuch für alle, die Gutes noch besser tun wollen. Phineo, Berlin

McKinsey Global Institute (2018) Skill shift; Automation and the future of the workforce. McKinsey\&Company. https://www.mckinsey.com/featured-insights/future-of-work/skill-shift-automationand-the-future-of-the-workforce

Meier C (2018a) KI-basierte Anwendungen für L\&D; Beispiele und Profilierungsoptionen für Learning Professionals. https://www.scil.ch/2018/10/01/ki-basierte-anwendungen-fuer-ld-beispiele-undprofilierungsoptionen-fuer-learning-professionals-clc-2018-kassel/

Meier C (2018b) scil Innovationskreis 2018: Smarte Personalentwicklungsstrategien im Zeitalter der digitalen Transformation. Universität St. Gallen / SCIL. https://www.scil-aktuell.ch/2018/03/ 
21/scil-innovationskreis-2018-smarte-personalentwicklungsstrategien-im-zeitalter-der-digitalentransformation/. Zugegriffen: 24. März 2019

Nedelkoska L, Quintini G (2018) Automation, skills use and training. OECD Publishing. https://doi.org/ 10.1787/2e2f4eea-en

Pulliam Phillips P, Phillips JJ (2007) The value of learning. How organisations capture value and ROI and translate it into support, improvement and funds. Pfeiffer, San Francisco

Seufert S, Guggemos J, Meier C, Helfritz KH (2018) Digitale Kompetenzen von Personalentwicklern; Digitale Reife und Augmentationsstrategien in der Personalentwicklung. Universität St.Gallen (IWPdbB, scil) / Deutsche Gesellschaft für Personalentwicklung, Frankfurt a.M. / St.Gallen

Thalheimer W (2018) The Learning-Transfer Evaluation Model; Sending messages to enable learning effectiveness. https://www.worklearning.com/wp-content/uploads/2018/02/Thalheimer-TheLearning-Transfer-Evaluation-Model-Report-for-LTEM-v12.pdf

Wahlster W (2017) Künstliche Intelligenz versus menschliche Intelligenz: Wie lernen, verstehen und denken Computer? Universität Mainz, Mainz

Wahlster W (2017) Künstliche Intelligenz als Treiber der zweiten Digitalisierungswelle. IM+io 2017:10-13 